PROCEEDINGS OF THE

AMERICAN MATHEMATICAL SOCIETY

Volume 132, Number 11, Pages 3141-3152

S 0002-9939(04)07565-3

Article electronically published on June 16, 2004

\title{
A MULTILINEAR GENERALISATION OF THE CAUCHY-SCHWARZ INEQUALITY
}

\author{
ANTHONY CARBERY
}

(Communicated by Andreas Seeger)

\begin{abstract}
We prove a multilinear inequality which in the bilinear case reduces to the Cauchy-Schwarz inequality. The inequality is combinatorial in nature and is closely related to one established by Katz and Tao in their work on dimensions of Kakeya sets. Although the inequality is "elementary" in essence, the proof given is genuinely analytical insofar as limiting procedures are employed. Extensive remarks are made to place the inequality in context.
\end{abstract}

\section{INTRODUCTION}

Let $X_{1}, \ldots, X_{n}$ be measure spaces, and let $K: X_{1} \times \ldots \times X_{n} \rightarrow \mathbb{R}$ be a nonnegative measurable function. Define, for $1 \leq j \leq n$,

$$
A_{j}\left(s_{j}\right)=\int K\left(x_{1}, x_{2}, \ldots, x_{j-1}, s_{j}, x_{j+1}, \ldots, x_{n}\right) d x_{1} \ldots \widehat{d x}_{j} \ldots d x_{n} .
$$

(We use simply $d x_{i}, d y_{i}$, etc. to denote integration on the measure space $X_{i}$, and the " $\wedge$ " signifies omission.) Also, for $1 \leq j \leq n-1$, define

$$
B_{j}\left(s_{j}, s_{j+1}\right)=\int K\left(x_{1}, x_{2}, \ldots, x_{j-1}, s_{j}, s_{j+1}, x_{j+2}, \ldots, x_{n}\right) d x_{1} d x_{2} \ldots \widehat{d x}_{j} \widehat{d x}_{j+1} \ldots d x_{n} .
$$

Finally, define the functional $Q_{n}$ by

$$
Q_{n}^{n+1}(K)=\int A_{1}\left(s_{1}\right) B_{1}\left(s_{1}, s_{2}\right) \ldots B_{n-1}\left(s_{n-1}, s_{n}\right) A_{n}\left(s_{n}\right) d s_{1} \ldots d s_{n} .
$$

It is more suggestive, but notationally more cumbersome, to write $Q_{n}^{n+1}(K)$ as

$$
\begin{gathered}
K\left(s_{1}, \quad x_{2}^{0}, \ldots, x_{j}^{0}, \ldots, x_{n}^{0}\right), \\
K\left(s_{1}, \quad s_{2}, \ldots, x_{j}^{1}, \ldots, x_{n}^{1}\right), \\
\vdots \\
K\left(x_{1}^{j}, \quad x_{2}^{j}, \ldots, s_{j}, s_{j+1}, \ldots, x_{n}^{j}\right), \\
\vdots \\
K\left(x_{1}^{n-1}, x_{2}^{n-1}, \ldots, s_{n-1}, s_{n}\right), \\
K\left(x_{1}^{n}, \quad x_{2}^{n}, \ldots, x_{n-1}^{n}, s_{n}\right),
\end{gathered}
$$

Received by the editors June 12, 2003.

2000 Mathematics Subject Classification. Primary 05A20, 42 B99. 
where the integration is performed over all visible variables. Briefly, with the $n(n+1)$ variables $x_{k}^{j} \quad(0 \leq j \leq n, 1 \leq k \leq n)$, we contract on the variables $x_{j+1}^{j}$ and $x_{j+1}^{j+1}$, i.e., we set $x_{j+1}^{j}=x_{j+1}^{j+1}=s_{j+1}, 0 \leq j \leq n-1$ before integrating.

Our multilinear generalisation of the Cauchy-Schwarz inequality is the following:

Theorem. With $K$ as above and $f_{j}: X_{j} \rightarrow \mathbb{R}$ nonnegative measurable functions, then

$$
\int K\left(x_{1}, \ldots, x_{n}\right) f_{1}\left(x_{1}\right) \ldots f_{n}\left(x_{n}\right) d x_{1} \ldots d x_{n} \leq Q_{n}(K)\left\|f_{1}\right\|_{n+1} \ldots\left\|f_{n}\right\|_{n+1} .
$$

Since when $n=1, Q_{1}(K)=\|K\|_{2}$, we recover the Cauchy-Schwarz inequality; when $n=2$ we obtain

$$
\begin{aligned}
& \int K\left(x_{1}, x_{2}\right) f_{1}\left(x_{1}\right) f_{2}\left(x_{2}\right) d x_{1} d x_{2} \\
\leq & \left(\int K(s, \alpha) K(s, t) K(\beta, t) d s d t d \alpha d \beta\right)^{\frac{1}{3}}\left\|f_{1}\right\|_{3}\left\|f_{2}\right\|_{3}
\end{aligned}
$$

(which is in fact also an elementary inequality). For extensive remarks on the significance of the functional $Q_{n}$, comparisons with more standard measures of size of $K$, and on the theorem itself, see Section 3 below.

The special case of the theorem corresponding to each $X_{i}$ being a probability space, and $f_{i} \equiv 1$ on $X_{i}$, was proved by elementary means in [C]. Upon chasing normalisations, this gives the theorem when each $f_{i}$ is the characteristic function of a measurable set; that is, if we replace the $L^{n+1}$ norms on the right-hand side by the Lorentz norms associated to $L^{n+1,1}$ (see, for example, [SW]). It was also shown in $[\mathrm{C}]$ that the theorem is true when any two consecutive $L^{n+1,1}$ spaces are replaced by their Lebesgue counterparts $L^{n+1}$. However, the method there did not yield the case of two "separated" $L^{n+1}$ spaces, still less the case of three or more $L^{n+1}$ spaces. This is what we achieve in this note.

The author would like to thank Bill Beckner for several helpful comments.

\section{Proof of Theorem}

For the reader's convenience, and also because it plays an important role in the full theorem, we first prove the special case when each $X_{i}$ is a probability space and $f_{i} \equiv 1$.

Lemma 1 ([C], see also [KT]). If $K: X_{1} \times \ldots \times X_{n} \rightarrow \mathbb{R}$ is a nonnegative measurable function, and each $X_{i}$ is a probability space, then

$$
\int K \leq Q_{n}(K)
$$

Proof. By homogeneity we may assume that $Q_{n}(K)=1$. Consider

$$
\int \frac{B_{1}\left(s_{1}, s_{2}\right) B_{2}\left(s_{2}, s_{3}\right) \ldots B_{n-1}\left(s_{n-1}, s_{n}\right)}{A_{2}\left(s_{2}\right) A_{3}\left(s_{3}\right) \ldots A_{n-1}\left(s_{n-1}\right)} d s_{1} \ldots d s_{n} .
$$

Performing the integration with respect to $s_{1}$ yields a factor $A_{2}\left(s_{2}\right)$, cancelling with the same factor in the denominator; similarly, integration with respect to $s_{2}, s_{3}$, etc. in turn, up to and including $s_{n-2}$, leaves one with

$$
\int B_{n-1}\left(s_{n-1}, s_{n}\right) d s_{n-1} d s_{n}=\int K
$$


Hence,

$$
\begin{gathered}
\int K=\int \frac{\prod_{i=1}^{n-1} B_{i}\left(s_{i}, s_{i+1}\right)}{\prod_{i=2}^{n-1} A_{i}\left(s_{i}\right)} d s_{1} \ldots d s_{n} \\
\leq \frac{1}{n+1} \int \frac{\prod_{i=1}^{n-1} B_{i}\left(s_{i}, s_{i+1}\right)}{\prod_{i=2}^{n-1} A_{i}\left(s_{i}\right)}\left\{A_{1}\left(s_{1}\right) \ldots A_{n}\left(s_{n}\right)+\frac{1}{A_{1}\left(s_{1}\right)}+\ldots+\frac{1}{A_{n}\left(s_{n}\right)}\right\} d s_{1} \ldots d s_{n}
\end{gathered}
$$

(by the geometric-arithmetic mean inequality)

$$
:=\frac{1}{n+1}\left\{I_{0}+I_{1} \ldots+I_{n}\right\} \text {. }
$$

Now $I_{0}=Q_{n}(K)^{n+1}=1$. To calculate $I_{n}$ observe that it is the same as (1) with an extra $A_{n}\left(s_{n}\right)$ in the denominator. Thus,

$$
I_{n}=\int \frac{B_{n-1}\left(s_{n-1}, s_{n}\right)}{A_{n}\left(s_{n}\right)} d s_{n-1} d s_{n}=\int 1 d s_{n}=1
$$

since $X_{n}$ is a probability space.

To calculate $I_{1}$ observe that it is the same as (1) with an extra $A_{1}\left(s_{1}\right)$ in the denominator. Thus, proceeding as in the evaluation of (1) but with the ordering $s_{n}, s_{n-1}, \ldots$, we see that

$$
I_{1}=\int \frac{B_{1}\left(s_{1}, s_{n}\right)}{A_{1}\left(s_{1}\right)} d s_{2} d s_{1}=\int 1 d s_{1}=1 .
$$

Finally, to calculate $I_{j}$, which is the same as (1) with the factor $A_{j}\left(s_{j}\right)$ in the denominator replaced by $A_{j}\left(s_{j}\right)^{2}$, we proceed as in the evaluation of $(1)$, arriving at

$$
\begin{aligned}
& \int \frac{B_{j-1}\left(s_{j-1}, s_{j}\right) \ldots B_{n-1}\left(s_{n-1}, s_{n}\right)}{A_{j}\left(s_{j}\right)^{2} A_{j+1}\left(s_{j+1}\right) \ldots A_{n-1}\left(s_{n-1}\right)} d s_{j-1} \ldots d s_{n} \\
= & \int \frac{B_{j-1}\left(s_{j-1}, s_{j}\right) \ldots B_{n-1}\left(s_{n-1}, s_{n}\right)}{A_{j}\left(s_{j}\right)^{2} A_{j+1}\left(s_{j+1}\right) \ldots A_{n-1}\left(s_{n-1}\right)} d s_{n} \ldots d s_{j-1} \\
= & \int \frac{B_{j-1}\left(s_{j-1}, s_{j}\right) B_{j}\left(s_{j}, s_{j+1}\right)}{A_{j}\left(s_{j}\right)^{2}} d s_{j+1} d s_{j} d s_{j-1} \\
= & \int A_{j}\left(s_{j}\right)^{2} / A_{j}\left(s_{j}\right)^{2} d s_{j}=1 .
\end{aligned}
$$

Thus, $I_{j}=1$ for $0 \leq j \leq n$, and so $\int K \leq 1=Q_{n}(K)$ as required.

Corollary. If $X_{1}, \ldots, X_{n}$ are general measure spaces and $E_{1}, \ldots, E_{n}$ are measurable subsets of $X_{1}, \ldots, X_{n}$ respectively, then

$$
\int K\left(x_{1}, \ldots, x_{n}\right) \chi_{E_{1}}\left(x_{1}\right) \ldots \chi_{E_{n}}\left(x_{n}\right) d x_{1} \ldots d x_{n} \leq Q_{n}(K)\left|E_{1}\right|^{1 /(n+1)} \ldots\left|E_{n}\right|^{1 /(n+1)},
$$

where $|\cdot|$ denotes measure on $X_{i}$.

Proof. Apply Lemma 1, taking $X_{i}=E_{i}$ with normalised measure. 
Lemma 2. Suppose $f_{i}: X_{i} \rightarrow \mathbb{R}$ is a simple function, and that $f_{i} \geq 1$ on $\operatorname{supp} f_{i}$. Then

$$
\begin{aligned}
& \int K\left(x_{1}, \ldots, x_{n}\right) f_{1}\left(x_{1}\right) \ldots f_{n}\left(x_{n}\right) d x_{1} \ldots d x_{n} \\
\leq & C_{n} Q_{n}(K) \prod_{i=1}^{n}\left\|f_{i}\right\|_{n+1} \prod_{i=1}^{n}\left(\log \left[2\left\|f_{i}\right\|_{\infty}\right]\right)^{\frac{n}{n+1}}
\end{aligned}
$$

(where $C_{n}$ depends only on $n$ ).

Proof. Let $E_{k}^{i}=\left\{x \in X_{i} \mid 2^{k-1} \leq f_{i}(x)<2^{k}\right\}$. Then $f_{i}(x) \leq \sum_{k=1}^{\infty} 2^{k} \chi_{E_{k}^{i}}(x)$. So

$$
\begin{aligned}
& \int K(x) \prod_{i=1}^{n} f_{i}\left(x_{i}\right) d x_{i} \\
\leq & \sum_{k_{1}, \ldots, k_{n=1}}^{\infty} 2^{k_{1}} \ldots 2^{k_{n}} \int K(x) \prod_{i=1}^{n} \chi_{E_{k_{i}}^{i}}\left(x_{i}\right) d x_{i} \\
\leq & \sum_{k_{1}, \ldots, k_{n=1}}^{\infty} 2^{k_{1}} \ldots 2^{k_{n}} Q_{n}(K) \prod_{i=1}^{n}\left|E_{k_{i}}^{i}\right|^{\frac{1}{n+1}}
\end{aligned}
$$

(by the Corollary)

$$
=Q_{n}(K) \prod_{i=1}^{n}\left(\sum_{k=1}^{\infty} 2^{k}\left|E_{k}^{i}\right|^{\frac{1}{n+1}}\right) .
$$

Now

$$
\sum_{k=1}^{M} 2^{k}\left|E_{k}^{i}\right|^{\frac{1}{n+1}} \leq\left(\sum_{k=1}^{M} 2^{k(n+1)}\left|E_{k}^{i}\right|\right)^{\frac{1}{n+1}} M^{\frac{n}{n+1}}
$$

and so

$$
\sum_{k=1}^{\infty} 2^{k}\left|E_{k}^{i}\right|^{\frac{1}{n+1}} \leq C_{n}\left\|f_{i}\right\|_{n+1}\left(\log \left[2\left\|f_{i}\right\|_{\infty}\right]\right)^{\frac{n}{n+1}}
$$

yielding the result.

Lemma 2 looks like a fatally flawed version of the theorem we wish to prove; nonetheless, as we shall now see, in the presence of product structure, the situation can be rescued. We may continue to assume, without loss of generality, that each $f_{i}$ is simple, and that $f_{i} \geq 1$ on $\operatorname{supp} f_{i}$ (otherwise multiply through by suitable constants).

Let $\mathbf{x}_{i}=\left(x_{i}^{1}, \ldots, x_{i}^{m}\right) \in X_{i} \times \ldots \times X_{i}$ for a suitable $m \in \mathbb{N}$. Let

$$
F_{i}\left(\mathbf{x}_{i}\right)=f_{i}\left(x_{i}^{1}\right) \ldots f_{i}\left(x_{i}^{m}\right)=f_{i} \otimes \ldots \otimes f_{i}\left(\mathbf{x}_{i}\right),
$$

and let

$$
\kappa\left(\mathbf{x}_{1}, \ldots, \mathbf{x}_{n}\right)=K \otimes \ldots \otimes K\left(\mathbf{x}_{1}, \ldots, \mathbf{x}_{n}\right) .
$$


Then, trivially, $Q_{n}(\kappa)=Q_{n}(K)^{m}$ and $\left\|F_{i}\right\|_{p}=\left\|f_{i}\right\|_{p}^{m}$. By Lemma 2,

$$
\begin{aligned}
& \int \kappa\left(\mathbf{x}_{1}, \ldots, \mathbf{x}_{n}\right) \prod_{i=1}^{n} F_{i}\left(\mathbf{x}_{i}\right) d \mathbf{x}_{i} \\
\leq & C_{n} Q_{n}(\kappa) \prod_{i=1}^{n}\left\|F_{i}\right\|_{n+1} \prod_{i=1}^{n}\left(\log \left[2\left\|F_{i}\right\|_{\infty}\right]\right)^{\frac{n}{n+1}} \\
= & C_{n} Q_{n}(K)^{m} \prod_{i=1}^{n}\left\|f_{i}\right\|_{n+1}^{m} \prod_{i=1}^{n}\left(m \log \left[2\left\|f_{i}\right\|_{\infty}\right]\right)^{\frac{n}{n+1}} \\
= & C_{n} m^{\frac{n^{2}}{n+1}}\left\{Q_{n}(K) \prod_{i=1}^{n}\left\|f_{i}\right\|_{n+1}\right\}^{m}\left(\prod_{i=1}^{n} \log \left[2\left\|f_{i}\right\|_{\infty}\right]\right)^{\frac{n}{n+1}} .
\end{aligned}
$$

Hence,

$$
\begin{aligned}
& \int K\left(x_{1}, \ldots, x_{n}\right) \prod_{i=1}^{n} f_{i}\left(x_{i}\right) d x_{i} \\
= & \left\{\int \kappa\left(\mathbf{x}_{1}, \ldots, \mathbf{x}_{n}\right) \prod_{i=1}^{n} F_{i}\left(\mathbf{x}_{i}\right) d \mathbf{x}_{i}\right\}^{\frac{1}{m}} \\
\leq & C_{n}^{\frac{1}{m}} m^{\frac{n^{2}}{m(n+1)}} Q_{n}(K) \prod_{i=1}^{n}\left\|f_{i}\right\|_{n+1}\left\{\prod_{i=1}^{n} \log \left[2\left\|f_{i}\right\|_{\infty}\right]\right\}^{\frac{n}{m(n+1)}} .
\end{aligned}
$$

As $m \rightarrow \infty, C_{n}^{\frac{1}{m}} \rightarrow 1, m^{\frac{n^{2}}{m(n+1)}} \rightarrow 1$ and $\left(\log \left[2\left\|f_{i}\right\|_{\infty}\right]\right)^{\frac{n}{m(n+1)}} \rightarrow 1$. Thus,

$$
\begin{aligned}
& \int K\left(x_{1}, \ldots, x_{n}\right) f_{i}\left(x_{i}\right) \ldots f_{n}\left(x_{n}\right) d x_{1} \ldots d x_{n} \\
\leq & Q_{n}(K)\left\|f_{1}\right\|_{n+1} \ldots\left\|f_{n}\right\|_{n+1},
\end{aligned}
$$

as desired.

\section{REMARKS}

1. This work grew out of an attempt to understand a certain combinatorial lemma employed by Katz and Tao, $\overline{\mathrm{KT}}$, in their study of estimates for the dimensions of Kakeya sets in $\mathbb{R}^{n}$. Their lemma is as follows, and is (essentially) equivalent (see [C]) to our Lemma 1.

Lemma $([\overline{\mathrm{KT}}])$. Let $X$ and $A_{1}, \ldots, A_{n}$ be finite sets and $g_{j}: X \rightarrow A_{j}$. Then

$$
\#\left\{\left(x_{0}, \ldots, x_{n}\right) \in X^{n+1} \mid g_{i}\left(x_{i-1}\right)=g_{i}\left(x_{i}\right), 1 \leq i \leq n\right\} \geq \frac{(\# X)^{n+1}}{\# A_{1} \ldots \# A_{n}} .
$$

Thus one sees the "contraction along the main and subdiagonal" feature of the definition of $Q(K)$ as a reflection of the formulation of the Katz-Tao lemma, which had a specific application in mind. Of course there are many potential combinatorial questions and inequalities similar to the one above, each with its own "integral" formulation as a multilinear form on a product of $L^{p}$-spaces. See Remark 3.8 below.

2. The lemma of Katz and Tao, although a statement about a finite number of functions between finite sets, also used product structure in its proof [KT]. The argument of Lemma 1 above gives an "elementary" alternative since it uses only the geometric-arithmetic mean inequality. However, when $n \geq 3$, the proof of the theorem at present needs the product structure: the author knows of no proof, save by enumeration of cases, even of the special case of the theorem where $X_{1}=X_{2}=$ $X_{3}$ is a 2 -point probability space and $K$ and $f_{1}, f_{2}, f_{3}$ take values in $\{1,2, \ldots 10\}$. It 
would seem to be an interesting challenge for the automatic theorem provers to give a direct and elementary proof of the theorem in this case.

3. The gist of the argument presented above is that if a general nonnegative multilinear form on $L^{p}$-spaces possesses a structure that is preserved under tensor products, then the inequality on probability spaces with each $f_{i} \equiv 1$ (equivalently on Lorentz-spaces) automatically self-improves to Lebesgue spaces $L^{p}$, with the same constant. (Lemma 2 says that $\|f\|_{p, 1} \leq C_{p}\|f\|_{p}\left(\log \left[2\|f\|_{\infty}\right]\right)^{\frac{1}{p^{\prime}}}$ if $f \geq 1$, and the product structure allows us to kill the $C_{p}$ and $\log \left\|f_{i}\right\|_{\infty}$ terms.) The $L^{p}$ norms are stable under tensor product in the sense that $\left\|f^{\otimes N}\right\|_{p, q}^{\frac{1}{N}} \rightarrow\|f\|_{p}$ as $N \rightarrow \infty, 1 \leq$ $q \leq \infty$. For examples see Remark 3.5 below.

4. Of course exploitation of product structure has a long history in analysis, especially in Functional Analysis (the spectral radius formula in Banach Algebras comes immediately to mind) and Complex Analysis but also in Harmonic Analysis (the Cotlar-Stein lemma and similar "almost-orthogonality" arguments, and the study of the Young and Hausdorff-Young inequalities [Be1] and more recent work by Beckner ( $\mathrm{Be} 2, \mathrm{Be} 3, \mathrm{Be} 4]))$. It may already have been noticed by the experts that Lorentz space inequalities exhibiting a product structure automatically improve to $L^{p}$-estimates: the argument of $[\mathrm{BL}$, pp. 155-156] is rather similar to the one we have presented here. (In connection with the Cotlar-Stein lemma, it is an amusing exercise to show that if $S$ and $T$ are bounded operators on a Hilbert space with $\|S\|,\|T\| \leq 1$ and $S T^{*}=S^{*} T=0$, then $\|S+T\| \leq 2^{\frac{1}{2}}, 2^{\frac{1}{4}}, \ldots, 1$ by taking products successively.)

5. (a) Let $X$ be a measure space, $E_{1}, \ldots, E_{n} \subseteq X$. Since obviously we have

$$
\int \chi_{E_{1} \ldots \chi_{E_{n}}}=\left|E_{1} \cap \ldots \cap E_{n}\right| \leq\left|E_{1}\right|^{\alpha_{1}} \ldots\left|E_{n}\right|^{\alpha_{n}}
$$

when $0 \leq \alpha_{i} \leq 1$ and $\alpha_{1}+\ldots+\alpha_{n}=1$, and since this inequality possesses product structure, we immediately deduce Hölder's inequality

$$
\int f_{1}(x) \ldots f_{n}(x) d x \leq\left\|f_{1}\right\|_{p_{1}} \ldots\left\|f_{n}\right\|_{p_{n}}
$$

where $\frac{1}{p_{1}}+\ldots+\frac{1}{p_{n}}=1,1 \leq p_{i} \leq \infty$.

(b) Let $X_{1}, \ldots, X_{n}$ be measure spaces and let $A_{i} \subseteq X_{1} \times \ldots \times \hat{X}_{i} \times \ldots \times X_{n}$. Then the Loomis-Whitney inequality

$$
\int \prod_{i=1}^{n} \chi_{A_{i}}\left(x_{1}, \ldots, \hat{x}_{i}, \ldots, x_{n}\right) d x_{i} \leq \prod_{i=1}^{n}\left|A_{i}\right|^{\frac{1}{n-1}}
$$

possesses product structure, and so we automatically obtain

$$
\int \prod_{i=1}^{n} f_{i}\left(x_{1}, \ldots, \hat{x}_{i}, \ldots, x_{n}\right) d x_{i} \leq \prod_{i=1}^{n}\left\|f_{i}\right\|_{n-1}
$$

for $f_{i}$ defined on $X_{1} \times \ldots \times \hat{X}_{i} \times \ldots \times X_{n}$. If now $g$ is defined on $X_{1} \times \ldots \times X_{n}$ and $P_{i} g\left(x_{1}, \ldots, \hat{x}_{i}, \ldots, x_{n}\right):=\sup _{x_{i}}\left|g\left(x_{1}, \ldots, x_{n}\right)\right|$, we have $g^{\frac{n}{n-1}} \leq \prod_{i=1}^{n} P_{i}\left(g^{\frac{1}{n-1}}\right)$, and so 
applying (3) we get

$$
\begin{aligned}
\int g^{\frac{n}{n-1}} & \leq \prod_{i=1}^{n}\left\|P_{i}\left(g^{\frac{1}{n-1}}\right)\right\|_{n-1} \\
& =\prod_{i=1}^{n}\left\|P_{i} g\right\|_{1}^{\frac{1}{n-1}}
\end{aligned}
$$

Thus

$$
\|g\|_{\frac{n}{n-1}} \leq \prod_{i=1}^{n}\left\|P_{i} g\right\|_{1}^{\frac{1}{n}}
$$

which, if $g \in C_{c}^{1}\left(\mathbb{R}^{n}\right)$, can in turn be dominated by $\prod_{i=1}^{n}\left\|\frac{\partial g}{\partial x_{i}}\right\|_{1}$, thus yielding the Gagliardo-Nirenberg inequality. Of course (2) (and likewise (3) and (4) directly) can be obtained by repeated use of Hölder's inequality.

(c) The best constant $A$ in Beckner's sharp Young's convolution inequality $($ Be1] $)$

$$
\int_{\mathbb{R}^{2 n}} f(x) g(x-y) h(y) d x d y \leq A\|f\|_{p_{1}}\|g\|_{p_{2}}\|h\|_{p_{3}}
$$

is given by $A=A_{p_{1} p_{2} p_{3}}^{n}:=\left(\prod_{i=1}^{3} p_{i}^{\frac{1}{p_{i}}} / p_{i}^{\prime \frac{1}{p_{i}^{\prime}}}\right)^{n}$ when $\frac{1}{p_{1}}+\frac{1}{p_{2}}+\frac{1}{p_{3}}=2$ (and if $\frac{1}{p_{1}}+\frac{1}{p_{2}}+\frac{1}{p_{3}} \neq 2$, the inequality fails.) This value for $A$ is obtained by testing (5) on (radial) Gaussians. We observe here that $A$ can also be obtained by testing (5) on characteristic functions of balls: that is, $A=D_{p_{1} p_{2} p_{3}}^{n}$, where

$$
D_{p_{1} p_{2} p_{3}}=\sup _{m} \sup _{a, b, c>0}\left\{\frac{\int_{\mathbb{R}^{2 m}} \chi_{\mathbb{B}}\left(\frac{x}{a}\right) \chi_{\mathbb{B}}\left(\frac{x-y}{b}\right) \chi_{\mathbb{B}}\left(\frac{y}{c}\right) d x d y}{\left\|\chi_{\mathbb{B}}(\dot{\bar{a}})\right\|_{p_{1}}\left\|\chi_{\mathbb{B}}(\dot{\bar{b}})\right\|_{p_{2}}\left\|\chi_{\mathbb{B}}(\dot{\bar{c}})\right\|_{p_{3}}}\right\}^{\frac{1}{m}}
$$

(and where $\mathbb{B}$ denotes the unit ball in $\mathbb{R}^{m}$ ). Indeed, in order to prove (5) with $A$ given by $D_{p_{1} p_{2} p_{3}}^{n}$ it suffices, by the product structure of the inequality, to prove it for characteristic functions of sets in all higher dimensions $m$; since we have the Hardy-Littlewood-Riesz-Sobolev rearrangement inequality

$$
\int_{\mathbb{R}^{2 m}} f(x) g(x-y) h(y) d x d y \leq \int_{\mathbb{R}^{2 m}} f^{*}(x) g^{*}(x-y) h^{*}(y) d x d y,
$$

it suffices to prove it for balls. (Here, $f^{*}$, etc. denotes the radial equimeasurable rearrangement of $f$, etc.) Thus the expression in (6), raised to the power $n$, gives an upper bound for $A$. On the other hand,

$$
A \geq \frac{\int_{\mathbb{R}^{2 n}} \chi_{\mathbb{B}}\left(\frac{x}{a}\right) \chi_{\mathbb{B}}\left(\frac{x-y}{b}\right) \chi_{\mathbb{B}}\left(\frac{y}{c}\right) d x d y}{\left\|\chi_{\mathbb{B}}(\dot{\dot{a}})\right\|_{p_{1}}\left\|\chi_{\mathbb{B}}(\dot{\bar{b}})\right\|_{p_{2}}\left\|\chi_{\mathbb{B}}(\dot{\bar{c}})\right\|_{p_{3}}}
$$

and since it is easy to see that $A$, as a function of $n$, is given by $A(n)=A(1)^{n}$, we obtain $A(1) \geq D_{p_{1}, p_{2}, p_{3}}$. Hence $A=D_{p_{1}, p_{2}, p_{3}}^{n}$.

Finally, one can relate directly $D_{p_{1}, p_{2}, p_{3}}$ to the constant $A_{p_{1}, p_{2}, p_{3}}$ obtained by testing on radial Gaussians: clearly $A_{p_{1}, p_{2}, p_{3}} \leq D_{p_{1}, p_{2}, p_{3}}$, and the reverse inequality can be obtained once again by a product argument and domination of characteristic functions of balls by Gaussians. See [BL, especially Proposition 3 on p. 155 . 
(d) While the general version of the Brascamp-Lieb inequality in $[\mathrm{L}$ does not seem accessible to these methods, nevertheless a restricted version that contains Beckner's Young's inequality, does follow as in (c). Let $\alpha_{1}, \ldots, \alpha_{M} \in \mathbb{R}^{k}$, with $k, M$ fixed and set, for $n \in \mathbb{N}$,

$$
\Lambda_{n}\left(f_{1}, \ldots, f_{M}\right)=\int_{\mathbb{R}^{n} \times \ldots \times \mathbb{R}^{n}} \prod_{i=1}^{M} f_{i}\left(\alpha_{i} \cdot x\right) d x
$$

where, for $x=\left(x_{1}, \ldots, x_{k}\right) \in \mathbb{R}^{n} \times \ldots \times \mathbb{R}^{n}$ and $\beta=\left(\beta^{1}, \ldots, \beta^{k}\right) \in \mathbb{R}^{k}, \beta \cdot x:=$ $\beta^{1} x_{1}+\ldots+\beta^{k} x_{k} \in \mathbb{R}^{n}$. Then, the best constant in the inequality

$$
\Lambda_{n}\left(f_{1}, \ldots, f_{M}\right) \leq A\left\|f_{1}\right\|_{p_{1}} \ldots\left\|f_{M}\right\|_{p_{M}}
$$

is given by

$$
A=\sup _{m} \sup _{a_{1}, \ldots, a_{M}>0}\left\{\frac{\Lambda_{m}\left(\chi_{\mathbb{B}}\left(\frac{\dot{a}}{a_{1}}\right), \ldots, \chi_{\mathbb{B}}\left(\frac{\dot{a_{M}}}{a^{\prime}}\right)\right)}{\prod_{i=1}^{M}\left\|\chi_{\mathbb{B}}\left(\frac{\dot{\bar{a}}}{a_{i}}\right)\right\|_{L^{p_{i}\left(\mathbb{R}^{m}\right)}}}\right\}^{\frac{n}{m}}
$$

as well as by the more familiar testing over Gaussians. The inequality exhibits product structure, and the Brascamp-Lieb-Luttinger rearrangement inequality BLL] allows us to argue as in (c) above. Note that this method gives no information as to extremals.

\section{6 . Conditions for equality, $n \geq 2$}

The condition for equality in Lemma 1 is easily read off from the proof: it is that the $A_{j}\left(s_{j}\right)$ are all identically equal almost everywhere to $Q_{n}(K)$. When $n=2$, the proof given in [C] shows that, under the normalisation $Q(K)=\left\|f_{1}\right\|_{3}=\left\|f_{2}\right\|_{3}=$ 1 , we get strict inequality in the main theorem unless

$$
A_{1}\left(s_{1}\right) A_{2}\left(s_{2}\right)=\frac{f_{1}^{3}\left(s_{1}\right)}{A_{1}\left(s_{1}\right)}=\frac{f_{2}^{3}\left(s_{2}\right)}{A_{2}\left(s_{2}\right)}
$$

almost everywhere, which clearly forces $A_{1}, A_{2}, f_{1}$ and $f_{2}$ to be constant almost everywhere. Thus, in general, when $X_{1}$ and $X_{2}$ are finite measure spaces, $A_{1}\left(s_{1}\right)=$ $Q(K) \mu_{2}(X)^{\frac{1}{3}} / \mu_{1}(X)^{\frac{2}{3}}$ a.e., $A_{2}\left(s_{2}\right)=Q(K) \mu_{1}(X)^{\frac{1}{3}} / \mu_{2}\left(X_{2}\right)^{\frac{2}{3}}$ a.e. and $f_{1}$ and $f_{2}$ are constants a.e., we have equality; otherwise strict inequality. For $n \geq 3$ conditions similar to the above give equality, but we do not know if this is the only way in which equality may be achieved.

\section{Comparison of $Q(K)$ with other quantities}

Since

$$
\begin{aligned}
& \int K\left(x_{1}, \ldots, x_{n}\right) f_{1}\left(x_{1}\right) \ldots f_{n}\left(x_{n}\right) d x_{1} \ldots d x_{n} \\
& \leq\|K\|_{\frac{n+1}{n}}\left\|f_{1} \otimes \ldots \otimes f_{n}\right\|_{n+1} \\
& =\|K\|_{\frac{n+1}{n}}\left\|f_{1}\right\|_{n+1} \ldots\left\|f_{n}\right\|_{n+1},
\end{aligned}
$$

it is obviously useful to relate $Q_{n}(K)$ to the $L^{p}$-norms and to $\|K\|_{\frac{n+1}{n}}$ in particular. When $n=1$ they coincide. When $n=2$, we have

$$
Q_{2}\left(\chi_{E}\right) \leq\left\|\chi_{E}\right\|_{\frac{3}{2}}
$$


since

$$
\begin{aligned}
& \int \chi_{E}(s, \alpha) \chi_{E}(s, t) \chi_{E}(\beta, t) d s d t d \alpha d \beta \\
\leq & \int \chi_{E}(s, \alpha) \chi_{E}(\beta, t) d s d t d \alpha d \beta \\
= & |E|^{2} .
\end{aligned}
$$

More generally, when $n$ is even, $n \geq 4$, and we are on a product of probability spaces,

$$
\begin{aligned}
Q_{n}^{n+1}(K) & =\int A_{1}\left(s_{1}\right) B_{1}\left(s_{1}, s_{n}\right) \ldots B_{n-1}\left(s_{n-1}, s_{n}\right) A_{n}\left(s_{n}\right) d s_{1} \ldots d s_{n} \\
& \leq\left\|B_{1}\right\|_{\infty}\left\|B_{3}\right\|_{\infty} \ldots \int A_{1}\left(s_{1}\right) B_{2}\left(s_{2}, s_{3}\right) B_{4}\left(s_{4}, s_{5}\right) \ldots d s_{1} d s_{n} \\
& \leq\|K\|_{\infty}^{\frac{n}{2}}\|K\|_{1}^{\frac{n+2}{2}}
\end{aligned}
$$

so that $Q_{n}\left(\chi_{E}\right) \leq|E|^{\frac{n+2}{2(n+1)}}$. Note that $\frac{n+2}{2(n+1)}$ goes to $\frac{1}{2}$ as $n \rightarrow \infty$, and indeed we also have, for $\|K\|_{2}=1$, on a product of probability spaces,

$$
\begin{aligned}
Q_{n}^{n+1}(K) & =\int \mathcal{O E} \leq \frac{1}{2} \int\left(\mathcal{O}^{2}+\mathcal{E}^{2}\right) \\
& =\frac{1}{2}(1+1)=1=\|K\|_{2}^{n+1}
\end{aligned}
$$

where $\mathcal{O}, \mathcal{E}$ denote the product of the odd/even rows respectively appearing in the expression for $Q_{n}$.

Examples show that these conclusions are sharp. Let

$$
E=\bigcup_{j \text { odd }}\left\{x \in[0,1]^{n}|| x_{j} \mid \leq \varepsilon\right\} .
$$

Then $|E| \approx \varepsilon$. When $j$ is odd we have $A_{j}\left(s_{j}\right) \geq \chi_{\left\{\left|s_{j}\right| \leq \varepsilon\right\}}$ and $B_{j}\left(s_{j}, s_{j+1}\right) \geq$ $\chi_{\left\{\left|s_{j}\right| \leq \varepsilon\right\}}$, while for $j$ even we have $A_{j}\left(s_{j}\right) \geq \varepsilon$ and $B_{j}\left(s_{j}, s_{j+1}\right) \geq \chi_{\left\{\left|s_{j+1}\right| \leq \varepsilon\right\}}$. So

$$
\begin{aligned}
Q_{n}^{n+1}\left(\chi_{E}\right) & =\int_{[0,1]^{n}} A_{1}\left(s_{1}\right) B_{1}\left(s_{1}, s_{2}\right) B_{2}\left(s_{2}, s_{3}\right) \ldots A_{n}\left(s_{n}\right) d s_{1} \ldots d s_{n} \\
& \geq \int \chi_{\left\{\left|s_{1}\right| \leq \varepsilon\right\}} \chi_{\left\{\left|s_{3}\right| \leq \varepsilon\right\}} \ldots d s_{1} \ldots d s_{n} \\
& = \begin{cases}\varepsilon^{\frac{n+1}{2}} & n \text { odd }, \\
\varepsilon^{\frac{n+2}{2}} & n \text { even. }\end{cases}
\end{aligned}
$$

In this example we thus have $Q_{n}\left(\chi_{E}\right) \geq C|E|^{\frac{1}{2}}$ for $n$ odd and $Q_{n}\left(\chi_{E}\right) \geq$ $C|E|^{\frac{n+2}{2(n+1)}}$ for $n$ even; so the inequalities proved above are sharp. On the other hand, there are also examples to show that $Q_{n}\left(\chi_{E}\right)$ may be much smaller than $|E|^{\frac{n}{n+1}}$ : if $E$ is an $\varepsilon$-neighbourhood of the straight line joining $(0,0, \ldots, 0)$ to $(1,1, \ldots, 1)$ in $[0,1]^{n}$, then each $A_{j}\left(s_{j}\right)$ is essentially constant (and equal to $C \varepsilon^{n-1}$ ), and so we have equality (see 6 above) in $|E|=\int \chi_{E} \leq Q_{n}\left(\chi_{E}\right)$, which is therefore much smaller than $|E|^{\frac{n}{n+1}}$.

Thus, on a product of probability spaces, we have that the quantities $Q_{n}\left(\chi_{E}\right)$ and $|E|^{\frac{n}{n+1}}$ are incommensurable when $n \geq 3$, and so neither of the main theorem and 
(7) contains the other. When $n=2$ the main theorem is an essential improvement over (7), irrespective of whether the measure spaces are probability spaces or not. (Perhaps this has something to do with its utility as in [KT].)

\section{Further possible extensions}

One may represent the functional $Q_{n}$ by the $(n+1) \times n$ matrix

$$
\left(\begin{array}{cccc}
* & & & \\
* & * & & \\
& * & & \\
& & \ddots & \\
& & & * \\
& & & *
\end{array}\right)
$$

with the *'s on the main and subdiagonals denoting contraction on variables in each column separately. What about other arrangements of *'s?

(a) Let $\mathcal{P}$ be an $(m+1) \times n$ matrix with two ${ }^{*}$ 's in each column. Let

$$
Q_{\mathcal{P}}^{m+1}(K)=\int \begin{gathered}
K\left(x_{1}^{0}, \ldots, x_{1}^{n}\right) \\
\vdots \\
K\left(x_{1}^{m}, \ldots, x_{n}^{m}\right)
\end{gathered}
$$

where, in each column, the variables associated to a $*$ are contracted. Let $\mathcal{G}(\mathcal{P})$ be the graph of $\mathcal{P}$ defined as follows: $\mathcal{G}(\mathcal{P})$ has vertices $\{0,1, \ldots, m\}$ and there is an edge joining $j$ and $k$ iff there is a column of $\mathcal{P}$ with ${ }^{*}$ 's in the $j$ th and $k$ th places. Wisewell [W] has shown that

$$
\int K(x) \prod_{i=1}^{n} f_{i}\left(x_{i}\right) d x_{i} \leq Q_{\mathcal{P}}(K) \prod_{i=1}^{n}\left\|f_{i}\right\|_{m+1}
$$

if and only if $\mathcal{G}(\mathcal{P})$ is acyclic. In particular, for

$$
\mathcal{P}=\left(\begin{array}{lll}
* & & * \\
* & * & \\
& * & *
\end{array}\right)
$$

the graph is cyclic and the inequality fails. It then becomes interesting to obtain lower bounds, for (recall the language of Katz and Tao [KT]

$$
\#\left\{\left(x_{0}, x_{1}, x_{2}\right) \in X^{3} \mid g_{1}\left(x_{0}\right)=g_{1}\left(x_{1}\right), g_{2}\left(x_{1}\right)=g_{2}\left(x_{2}\right), g_{3}\left(x_{2}\right)=g_{3}\left(x_{0}\right)\right\}
$$

where $g_{j}: X \rightarrow\{1, \ldots, N\}$ are functions and $X$ is a finite set. By the case $n=2$ of [KT], or the theorem, one has a lower bound of $(\# X)^{3} / N^{4}$, while the lower bound $(\# X)^{3} / N^{3}$ fails. In fact, if $(\# X)^{3} / N^{\alpha}$ is a lower bound, then $\alpha>3$ ([W]). What is the best $\alpha$ ?

(b) In connection with Hölder's inequality (with integral values of the exponent) one may consider placing three or more *'s in each column of $\mathcal{P}$. Thus, for $1 \leq j \leq$ $n$ let $B_{j} \subseteq\{0, \ldots, m\}$, and define $\mathcal{P}$ by placing *'s in the $j$ th column of $\mathcal{P}$ in the places indicated by $B_{j}$. Define $Q_{\mathcal{P}}(K)$ in the analogous way. One may then ask about the inequality

$$
\int K(x) \prod_{i=1}^{n} f_{i}\left(x_{i}\right) d x_{i} \leq Q_{\mathcal{P}}(K) \prod_{i=1}^{n}\left\|f_{i}\right\|_{\frac{m+1}{\# B_{i}-1}} .
$$

We define a "move" to be an operation transforming an $(m+1) \times q$ matrix of blanks and *'s into an $(m+1) \times(q+1)$ matrix of blanks and *'s by taking one 
column and forming an extra column to its right by shifting some of the ${ }^{*}$ 's across to the new column. If $\mathcal{P}$ can be transformed by a sequence of moves to a matrix $\mathcal{P}^{\prime}$ with exactly two ${ }^{*}$ 's in each column, with $\mathcal{G}\left(\mathcal{P}^{\prime}\right)$ acyclic, then $(8)$ holds, W]. If (8) holds, must there exist such a sequence of moves?

(c) Finally, we may think of the *'s as being black (the blanks white). What about multicoloured arrangements of ${ }^{*}$ 's? In this case, in defining $Q_{\mathcal{P}}$, we contract separately (within each column) over ${ }^{*}$ 's of the same colour. Thus

$$
\mathcal{P}_{1}=\left(\begin{array}{cc}
* & * \\
* & \cdot \\
\cdot & \cdot \\
\cdot & *
\end{array}\right)
$$

corresponds to

$$
Q_{\mathcal{P}_{1}}(K)=\left(\begin{array}{l}
K(s, t) \\
K(s, u) \\
K(v, u) \\
K(v, t)
\end{array} \quad d s d t d u d v\right)^{\frac{1}{4}}
$$

and

$$
\mathcal{P}_{2}=\left(\begin{array}{ccc}
* & * & \\
* & & * \\
\cdot & & * \\
\cdot & * &
\end{array}\right)
$$

corresponds to

$$
Q_{\mathcal{P}_{2}}(K)=\left(\begin{array}{l}
K(s, t, \alpha) \\
K(s, \beta, u) \\
K(v, \gamma, u) \\
K(v, t, \delta)
\end{array} \quad d s d t d u d v d \alpha d \beta d \gamma d \delta\right)^{\frac{1}{4}}
$$

In general, if $\mathcal{P}$ is an $(m+1) \times n$ matrix with multicoloured ${ }^{*}$ 's, let $\gamma_{j C}$ be the number of ${ }^{*}$ 's of colour $C$ occurring in the $j$ th column of $\mathcal{P}$, and let $\gamma_{j}=$ $\sum_{C}\left(\gamma_{j C}-1\right)$. The question is then whether

$$
\int K(x) \prod_{i} f_{i}\left(x_{i}\right) d x_{i} \leq Q_{\mathcal{P}}(K) \prod_{i=1}^{n}\left\|f_{i}\right\|_{\frac{m+1}{\gamma_{i}}} .
$$

Thus, for $\mathcal{P}_{1}$ above, (9) becomes

$$
\int K(x) f_{1}\left(x_{1}\right) f_{2}\left(x_{2}\right) d x_{1} d x_{2} \leq Q_{\mathcal{P}_{1}}(K)\left\|f_{1}\right\|_{2}\left\|f_{2}\right\|_{2}
$$

and for $\mathcal{P}_{2},(9)$ becomes

$$
\int K(x) f_{1}\left(x_{1}\right) f_{2}\left(x_{2}\right) f_{3}\left(x_{3}\right) d x_{1} d x_{2} d x_{3} \leq Q_{\mathcal{P}_{2}}(K)\left\|f_{1}\right\|_{2}\left\|f_{2}\right\|_{4}\left\|f_{3}\right\|_{4} .
$$

Both (10) and (11) are true, and in fact (10) follows from (11) via the move transforming $\mathcal{P}_{1}$ to $\mathcal{P}_{2}$. Since $Q_{\mathcal{P}_{1}}(K) \leq\|K\|_{2}$, (10) is a strengthening of the Hilbert-Schmidt criterion for boundedness on $L^{2}$ of a bilinear form. The proofs of (10) and (11) are elementary. In the language of Katz and Tao, (10) amounts to counting rectangles and (11) to counting quadrilaterals with two sides parallel. See also $\mathrm{MT}$. 


\section{REFERENCES}

[Be1] W. Beckner, Inequalities in Fourier Analysis, Annals of Math. 102 (1975) 159-182. MR 52:6317

[Be2] W. Beckner, Geometric inequalities in Fourier Analysis, in Essays on Fourier Analysis in Honor of E.M. Stein, ed. C. Fefferman, R. Fefferman, S. Wainger, Princeton U. Press (1995) 36-68. MR 95m:42004

[Be3] W. Beckner, Sharp inequalities and geometric manifolds, J. Fourier Anal. Appl. 3 (special issue) (1997) 825-836. MR 2000c:58059

[Be4] W. Beckner, Geometric asymptotics and the logarithmic Sobolev inequality, Forum Math. 11 (1999) 105-137. MR 2000a:46049

[BL] H.J. Brascamp and E. Lieb, Best constants in Young's inequality, its converse, and its generalization to more than three functions, Adv. Math. 20 (1976) 151-173. MR 54:492

[BLL] H.J. Brascamp, E. Lieb and J.M. Luttinger, A general rearrangement inequality for multiple integrals, Jour. Funct. Anal. 17 (1974) 227-237. MR 49:10835

[C] A. Carbery, A remark on an inequality of Katz and Tao, in Harmonic Analysis at Mount Holyoke, eds. W. Beckner, A. Nagel, A. Seeger and H. Smith, Contemporary Mathematics 320, Amer. Math. Soc. (2003) 71-75.

[KT] N. Katz and T. Tao, Bounds on arithmetic projections, and applications to the Kakeya conjecture, Math. Res. Lett. 6 (1999), 625-633. MR 2000m:28006

[L] E. Lieb, Gaussian kernels have only Gaussian maximizers, Inventiones Math. 102 (1990) 179-208. MR 91i:42014

[MT] G. Mockenhaupt and T. Tao, Restriction and Kakeya phenomena for finite fields, Duke Math. J. 121 (2004), 35-74.

[SW] E.M. Stein and G. Weiss, An Introduction to Fourier Analysis on Euclidean spaces, Princeton U. Press, Princeton (1971) MR 46:4102

[W] L. Wisewell, personal communication.

School of Mathematics, University of Edinburgh, James Clerk Maxwell Building, King's Buildings, Edinburgh EH9 3JZ, United Kingdom

E-mail address: A.Carbery@ed.ac.uk 